Return to the Manage Active Submissions page at http://spie.org/submissions/tasks.aspx and approve or disapprove this submission. Your manuscript will not be published without this approval. Please contact author_help@spie.org with any questions or concerns.

\title{
Damage severity assessment in wind turbine blade laboratory model through fuzzy finite element model updating
}

\author{
Heather Turnbull \& Piotr Omenzetter \\ The Lloyd's Register Foundation Centre for Safety and Reliability Engineering, The University of \\ Aberdeen, Aberdeen AB24 3UE, UK
}

\begin{abstract}
The recent shift towards development of clean, sustainable energy sources has provided a new challenge in terms of structural safety and reliability: with aging, manufacturing defects, harsh environmental and operational conditions, and extreme events such as lightning strikes wind turbines can become damaged resulting in production losses and environmental degradation. To monitor the current structural state of the turbine, structural health monitoring (SHM) techniques would be beneficial. Physics based SHM in the form of calibration of a finite element model (FEMs) by inverse techniques is adopted in this research. Fuzzy finite element model updating (FFEMU) techniques for damage severity assessment of a small-scale wind turbine blade are discussed and implemented. The main advantage is the ability of FFEMU to account in a simple way for uncertainty within the problem of model updating. Uncertainty quantification techniques, such as fuzzy sets, enable a convenient mathematical representation of the various uncertainties. Experimental frequencies obtained from modal analysis on a small-scale wind turbine blade were described by fuzzy numbers to model measurement uncertainty. During this investigation, damage severity estimation was investigated through addition of small masses of varying magnitude to the trailing edge of the structure. This structural modification, intended to be in lieu of damage, enabled non-destructive experimental simulation of structural change. A numerical model was constructed with multiple variable additional masses simulated upon the blades trailing edge and used as updating parameters. Objective functions for updating were constructed and minimized using both particle swarm optimization algorithm and firefly algorithm. FFEMU was able to obtain a prediction of baseline material properties of the blade whilst also successfully predicting, with sufficient accuracy, a larger magnitude of structural alteration and its location.
\end{abstract}

Keywords: Structural health monitoring, damage detection, wind turbine blade, fuzzy finite element model updating, uncertainty quantification, particle swarm optimization, firefly algorithm

$\begin{array}{llll}\text { Abbreviations } & & \\ \text { EMA } & \text { Experimental modal analysis } & \text { LE } & \text { Leading edge } \\ \text { FA } & \text { Firefly algorithm } & \text { MCS } & \text { Monte Carlo simulation } \\ \text { FEM } & \text { Finite element model } & \text { NI } & \text { National Instruments } \\ \text { FEMU } & \text { Finite element model updating } & \text { O\&M } & \text { Operation and maintenance } \\ \text { FFEMU } & \text { Fuzzy finite element model updating } & \text { PSO } & \text { Particle swarm optimization } \\ \text { FRF } & \text { Frequency response function } & \text { SHM } & \text { Structural health monitoring } \\ \text { GOA } & \text { Global optimization algorithm } & \text { TE } & \text { Trailing edge }\end{array}$

\section{INTRODUCTION}

Advancement of technologies which harvest the earth's natural resources for power generation purposes have led to harnessing clean energy from sources such as solar, biomass, geothermal and wind. Developments in material technology and availability of resources have contributed to the significant growth, depicted in Figure 1, observed in the wind energy industry [1]. 
Return to the Manage Active Submissions page at http://spie.org/submissions/tasks.aspx and approve or disapprove this submission. Your manuscript will not be published without this approval. Please contact author_help@spie.org with any questions or concerns.

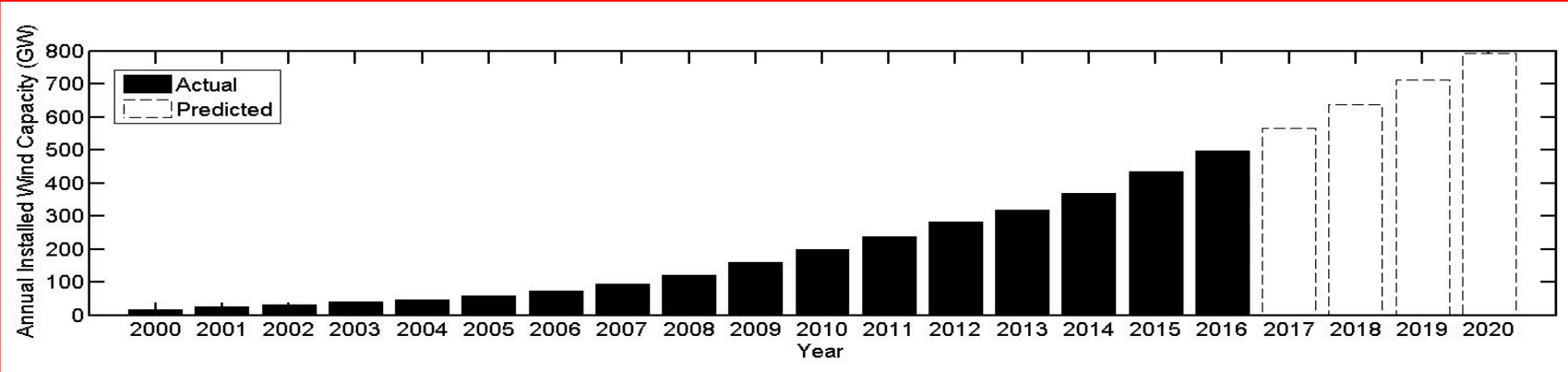

Figure 1 - Global annual installed wind capacity 2000-2020 [1]

Predictions from the global wind energy council forecast annual installed wind capacity to maintain a steady growth moving towards 2020 [1], therefore increasing cost effectiveness of this resource is of paramount importance. Costs associated with operation and maintenance (O\&M) of wind turbines can be significant, estimated at $20-30 \%$ of the lifecycle costs for onshore turbines and $30 \%$ of the higher lifecycle costs for their offshore counterparts [2]. In particular, blades are a crucial component in terms of economic factors, structural safety and energy production; however, materials and manufacture methods lead to an increased susceptibility to degradation from moisture absorption, leading edge erosion, fatigue and delamination. Current condition monitoring practices include visual inspections at predefined intervals, ultrasonic testing and acoustic emissions testing.

Although these methods are non-destructive, they are subjective due to dependence on the skill of the inspector and provide no information on the structural state between monitoring intervals. Techniques classified as structural health monitoring (SHM) have been developed to continuously monitor the health of a structure as opposed to evaluation of the structure at predefined intervals. The ability to continuously monitor a wind turbine will facilitate early prediction of damage, enabling maintenance to be scheduled at convenience and mitigations to be introduced before damage severity increases.

Physics based SHM techniques such as calibration of finite element models (FEM) by inverse techniques, known as finite element model updating (FEMU), have potential for damage severity assessment. Deterministic FEMU is an optimization problem where an objective function, containing the difference between experimental and analytical modal parameters, is minimized to provide an updated set of model parameters. Although these methods are beneficial in theory, idealization, discretization and model parameter errors are often encountered in FEMU and affect the integrity of the investigation [3]. Probabilistic and alternative uncertainty quantification methods can use uncertain measurement data and FEMs to propagate uncertainty into the updated parameters.

Probabilistic FEMU methods utilize knowledge of the random distribution of measured modal parameters to determine statistical indices of model parameters. A probabilistic Bayesian damage identification framework was investigated on an operational footbridge by Behmanesh and Moaveni $[4,5]$. The influence of continuously collected data on the probabilistic methodology was investigated and the results compared to a deterministic damage identification [4]. Further research by Behmanesh and Moaveni [5] utilized the Bayesian FEMU methodology for damage detection of the structure under varying environmental conditions. Within both studies, the authors simulated damage through addition of experimental mass to segments of the bridge and considered the added mass of each segment as updating parameters.

The link between probabilistic and fuzzy logic was investigated by Chandrashekhar and Ganguli [6] who used probabilistic methods for FEMU to update a cantilever beam exposed to varying damage extents. A probabilistic Monte Carlo simulation (MCS) was used to determine variation in a damage evaluation parameter (frequency) resulting from structural damage and material uncertainty. The obtained results were used as an input to a fuzzy logic system providing a visualization of the damage detected on the structure. This link was further developed by Simoen et al [7] who used both Bayesian and non-probabilistic (fuzzy) methods to model uncertainty in FEMU and successfully updated a reinforced concrete beam. Due to the fundamentally different nature of uncertainty modelling, the authors concluded that both approaches were appropriate, however, suitability of the method was found to be problem dependent.

Probabilistic methods are often dependent on specification of probability density functions to describe uncertain quantities. In construction of these, information is often unavailable or subject to engineering judgement and therefore the models created can be subjective. Alternative uncertainty quantification methods, such as fuzzy finite element model updating 


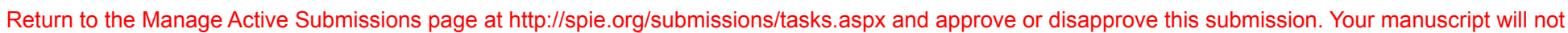
be published without this approval. Please contact author_help@spie.org with any questions or concerns.

(FFEMU) provide the benefit of not requiring any assumptions regarding the probability distribution of uncertain variables. FFEMU was discussed by Erdogan and Bakir [8] to investigate the effect of measurement noise on measured modal parameters. Global optimization algorithms (GOA) were utilized to determine the global optimum of the objective function at each fuzzy membership level with comparisons between membership functions of fuzzy updated parameters and results obtained via MCS methods. FFEMU was further investigated by Liu and Duan [9] for a full-scale bridge structure. This work considered the uncertain structural parameters as fuzzy variables, which led to fuzzification of the objective function for updating. Approaches reported in both [8] and [9] involve model updating of structures with data containing reducible uncertainty.

In this paper, the non-probabilistic FFEMU process is detailed and used for structural modification assessment of a smallscale wind turbine blade within laboratory conditions. This methodology enabled identification of the magnitude and location of structural modification whilst considering the uncertainty propagation in updated parameters. Two experimental campaigns, in baseline and altered states, were conducted to obtain the natural frequencies of the blade to be used as the target for updating studies. A numerical model of the blade was constructed with four added lumped mases, which were used as the updating parameters. Objective functions were constructed and minimized at each $\alpha$-level using both particle swarm optimization (PSO) algorithm and firefly algorithm (FA). Fuzzy updated parameters, which minimized the objective function at each fuzzy membership level predicted the location and magnitude of structural modification whilst accounting for uncertainty associated with these parameters.

\section{THEORY}

\subsection{Fuzzy sets and $\alpha$-level technique}

In classical set theory, the statement about an object belonging to a particular set is described in binary terms, providing sharply defined boundaries. In real life, however, for variables such as those used in structural models, this binary classification is idealized and no longer applicable due to the uncertainty associated with the variables. Fuzzy set method, developed by Zadeh [10], is a powerful mechanism to quantify uncertainty as it assigns each object with a degree of membership within a particular set through prescription of a membership function. For a fuzzy set $\tilde{A}$, the membership function is represented by $\mu_{\tilde{A}}(x)$ for all values of $x$ in the domain $X$ :

$$
\tilde{A}=\left\{\left(x, \mu_{\tilde{A}}(x)\right) \mid\left(x \in X, \mu_{\tilde{A}}(x) \in[0,1]\right)\right\}
$$

A membership function $\mu_{\tilde{A}}(x)$ value equal to one indicates that the element is a member of set $\tilde{A}$, while a value of zero indicates $x$ is undoubtedly not a member. A value between these two extremes indicates a degree of belief in the variable belonging to the set. The $\alpha$-level technique, often used in fuzzy arithmetic operations, involves decomposing the membership function of fuzzy variables into a number of levels, enabling a deterministic interval to be obtained for each level.

Deterministic FEMU can be considered as a constrained optimization problem in which the goal is to minimize an objective function containing the variation between analytical and experimental modal parameters. As this objective function is a function of the structural parameters, minimization leads to an updated set of structural parameters whose corresponding modal response parameters closely match the experimental response parameters.

\section{9 - 87 V. 5 (p.3 of 14) / Color: No / Format: A4 / Date: 4/11/2017 6:12:50 AM}


Return to the Manage Active Submissions page at http://spie.org/submissions/tasks.aspx and approve or disapprove this submission. Your manuscript will not be published without this approval. Please contact author_help@spie.org with any questions or concerns.

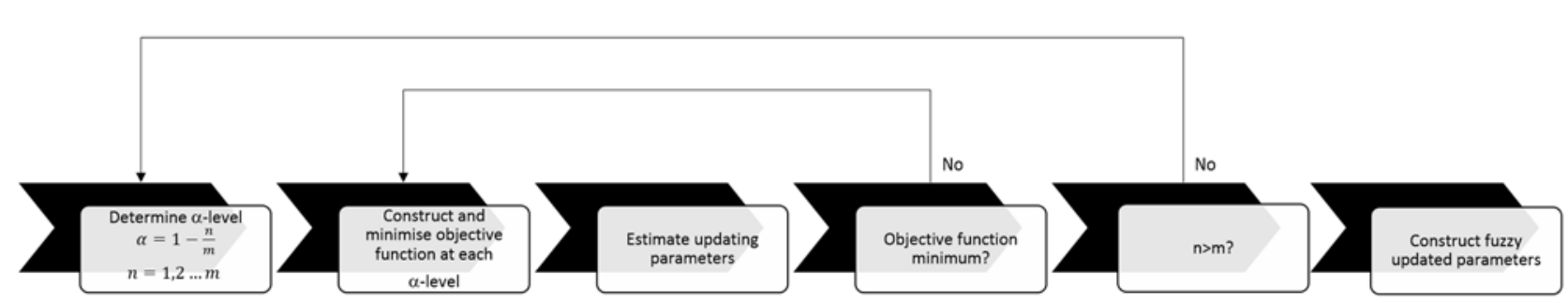

Figure 2 - FFEMU process

\subsection{Fuzzy finite element model updating}

\subsection{Theory}

FFEMU involves assigning membership functions values to the experimental responses obtained to introduce uncertainty, then propagation of this uncertainty through the model to calculate the uncertainty associated with the structural parameters. To do this, membership functions are divided into a number of levels $(\mathrm{m})$, an objective function is formulated at each of the $m$ levels and minimized to obtain the updated structural parameters at that level. Finally, the structural parameters at each level are then assembled to provide fuzzy updated parameters (Figure 2). The objective functions used for updating each of the levels are detailed in Section 2.4.

\subsection{Objective functions}

During FFEMU, two objective functions, a deterministic one and a fuzzy one, are required dependent on the level to be updated. The deterministic objective function, used for level $\alpha=1$, considering only frequency values for updating [11] is:

$$
f(\boldsymbol{\theta})=\sum_{i=1}^{n}\left[\left(\lambda_{i}^{a}(\boldsymbol{\theta})-\lambda_{i}^{e}\right) / \lambda_{i}^{e}(\boldsymbol{\theta})\right]^{2}
$$

where $\lambda$ represents eigenvalue (frequency), superscripts $a$ and $e$ refer to analytical and experimental values, respectively, and $n$ is the number of frequencies considered.

A fuzzy objective function, is required for subsequent $\alpha$-levels as these levels require the calculation of an interval updating parameter vector which minimizes the deviation between analytical and experimental modal parameters at the lower and upper bounds of the level respectively. A fuzzy objective function of this nature can be written as [8]:

$$
\begin{gathered}
f\left(\boldsymbol{\theta}^{I}\right)=\sum_{i=1}^{n}\left[\underline{\mathbf{r}}_{i}\left(\boldsymbol{\theta}^{I}\right) \mathbf{W}^{T} \underline{\mathbf{r}}_{i}\left(\boldsymbol{\theta}^{I}\right)+\overline{\mathbf{r}}_{i}\left(\boldsymbol{\theta}^{I}\right)^{T} \mathbf{W} \overline{\mathbf{r}}_{i}\left(\boldsymbol{\theta}^{I}\right)\right]^{2} \\
\underline{\mathbf{r}}_{i}\left(\boldsymbol{\theta}^{I}\right)=\left[\underline{\lambda}_{i}^{a}\left(\boldsymbol{\theta}^{I}\right)-\underline{\lambda}_{i}^{e}\right] / \underline{\lambda}_{i}^{e} \\
\overline{\mathbf{r}}_{i}\left(\boldsymbol{\theta}^{I}\right)=\left[\bar{\lambda}_{i}^{a}\left(\boldsymbol{\theta}^{I}\right)-\bar{\lambda}_{i}^{e}\right] / \bar{\lambda}_{i}^{e}
\end{gathered}
$$

where $\theta^{I}$ is the interval updating parameter vector, $\lambda_{i}{ }^{a}\left(\theta^{I}\right)$ and $\bar{\lambda}_{i}{ }^{a}\left(\theta^{I}\right)$ are the lower and upper values of analytical eigenvalue, respectively, whilst $\underline{\lambda}_{i}{ }^{e}$ and $\bar{\lambda}_{i}^{e}$ are the lower and upper values of experimental eigenvalues, respectively, and $\mathbf{W}$ is the weighting matrix. Variables $\underline{\mathbf{r}}_{i}$ and $\overline{\mathbf{r}}_{i}$ are relative modal frequency errors.

\subsection{Optimization algorithms}

A significant challenge in FFEMU is choosing a robust optimization algorithm capable of obtaining the global minimum solution of the objective function. Within the solution space, often local minimum solutions are known to exist which may cause the algorithm to converge at a non-optimum solution. In addition to this, the relationship between modal and structural parameters is generally non-linear in nature and therefore the objective function becomes complex. In this research, the applicability of two optimization algorithms, namely FA and PSO, was investigated. 
Return to the Manage Active Submissions page at http://spie.org/submissions/tasks.aspx and approve or disapprove this submission. Your manuscript will not be published without this approval. Please contact author_help@spie.org with any questions or concerns.

\subsection{Firefly algorithm}

The FA, developed by Yang [12], is a population based, meta-heuristic algorithm inspired by nature in particular the flashing behavior of fireflies. Meta-heuristic algorithms have the advantage of utilizing a combination of randomization and local searching to discover new solutions within the search space. Fireflies use bioluminescence, to create unique flashing patterns which can be used to attract mating partners, entice potential prey and as a deterring mechanism against potential predators [13].

FA is based on three fundamental assumptions:

1. Fireflies are unisex

2. Attractiveness of fireflies is proportional to their light intensity

3. The brightness of a firefly is determined by the calculated objective function value

Crucial to the success of FA is the definition of how light intensity varies and formulation of attractiveness. The light intensity, $I$, of the solution is proportional to the objective function value. The light intensity $I(r)$ decreases with distance from the source according to the following equation:

$$
I(r)=I_{o} e^{-\gamma r^{2}}
$$

where $I_{o}$ represents the intensity of light at the source, $r$ is the distance from the source and light absorption is modelled using the fixed light absorption coefficient, $\gamma,[13]$. As stated in the second assumption of the algorithm, attractiveness is directly proportional to their light intensity $I(r)$ and therefore attractiveness $\beta$ can be modelled as:

$$
\beta(r)=\beta_{0} e^{-\gamma r^{2}}
$$

where $\beta_{0}$ is the attractiveness at $r=0$. The distance between two fireflies $\mathbf{S}_{i}$ and $\mathbf{S}_{j}$ is calculated as the Euclidian distance:

$$
r_{i j}=\left\|\mathbf{S}_{i}-\mathbf{S}_{j}\right\|=\sqrt{\sum_{k=1}^{k=n}\left(S_{i k}-S_{j k}\right)^{2}}
$$

where $n$ denotes the dimensionality of the problem. To define the movement of the $i^{\text {th }}$ firefly towards the more attractive $i^{\text {th }}$ firefly the following equation is used:

$$
\mathbf{S}_{i}=\mathbf{S}_{i}+\beta_{o} e^{-\gamma r_{i j}^{2}}\left(\mathbf{S}_{j}-\mathbf{S}_{i}\right)+\alpha \boldsymbol{\varepsilon}_{i}
$$

where $\alpha$ is a randomization (mutation) parameter and $\varepsilon_{i}$ is a random vector chosen from a Gaussian distribution. This equation consists of three terms, the first indicating the current position of the $i^{\text {th }}$ firefly, the second controlling attraction towards a more attractive firefly whilst the third term is a randomization term [13].

\subsection{Particle swarm optimization}

The PSO algorithm is another metaheuristic optimization algorithm based on the way in which a group of animals such as a swarm of bees or school of fish search for the optimum feeding location. This nature-inspired algorithm developed by Kennedy and Eberhart [14], creates an initial swarm of particles in the solution space which are able to search for the optimum solution using their personal knowledge whilst also benefiting from the knowledge of the swarm. As a particle moves through the solution space, it has the ability to remember the individually achieved optimum value and the positional co-ordinates leading to this value. The particle also benefits from the knowledge of the entire swarm's optimum position and modifying its individual path towards the globally optimum solution.

In the PSO algorithm, the population of particles is randomly generated within the solution space with a random position $\mathbf{x}_{i}(t)$ and velocity $\mathbf{v}_{i}(t)$. The algorithm calculates an objective function value, often known as a cost value, of each particle using the specified objective function. The particles are influenced in their behavior by their individual best position $\operatorname{pbest}_{i}(t)$ and the optimum position of the swarm denoted as $\operatorname{gbest}(t)$. The position $\mathbf{x}_{i}(t)$ is updated in each iteration as follows:

$$
\mathbf{x}_{i}(t+1)=\mathbf{x}_{i}(t)+\mathbf{v}_{i}(t+1)
$$


Return to the Manage Active Submissions page at http://spie.org/submissions/tasks.aspx and approve or disapprove this submission. Your manuscript will not be published without this approval. Please contact author_help@spie.org with any questions or concerns.

where $i$ is the particle number and $t$ is the iteration number. The velocity of particles $\mathbf{v}_{i}(t)$ relative to the personal and global optimum solutions is updated at each iteration as follows:

$$
\mathbf{v}_{i}(t+1)=\gamma \times \mathbf{v}_{i}(t)+c_{1} \times \operatorname{rand}\left(\mathbf{p b e s t}_{i}(t)-\mathbf{x}_{\mathbf{i}}(t)\right)+c_{2} \times \operatorname{rand}\left(\mathbf{g b e s t}(t)-\mathbf{x}_{i}(t)\right)
$$

where $\mathbf{v}_{i}(t)$ and $\mathbf{v}_{i}(t+1)$ represent the initial and updated velocities respectively, $\gamma$ is the inertial weight, and $c_{1}$ and $c_{2}$ are the cognitive and social acceleration coefficients, respectively.

\section{PHYSICAL SYSTEM AND EXPERIMENTS}

Experimental modal analysis (EMA) was conducted on a small-scale wind turbine blade in laboratory conditions to obtain the modal parameters utilized in subsequent model calibration studies. This section introduces the experimental configuration of the blade and provides a description of the employed EMA techniques. A discussion of the experimental results from campaigns on the baseline and altered states of the blade is provided in this section. To model uncertainty in the updating parameters, uncertainty in the obtained modal parameters is modelled using the framework provided in Section 3.3.

\subsection{Experimental configuration}

The test specimen used within this research is a small-scale wind turbine blade from a Fortis turbine with a $5 \mathrm{~kW}$ rated power output and $5 \mathrm{~m}$ rotor diameter. The blade from a domestic scale turbine is manufactured from glass-fiber reinforced epoxy composite with a solid E387 airfoil profile cross section and $2.36 \mathrm{~m}$ length. Detailed material properties for this test specimen were unavailable from the manufacturer with known/measured properties of the blade shown in Table 1. A vertically orientated fixed-free configuration of the blade was chosen to simulate the support of an in-service turbine blade whilst minimizing lab space required. The root edge was clamped to a heavy base, located on a concrete floor to minimize the influence of uncontrolled excitation from the environment.

The dynamic response of the structure was investigated using EMA techniques, in particular a roving hammer methodology to calculate the modal parameters. The impact hammer, a Brüel \& Kjær 8206 instrument with a sensitivity of $1.14 \mathrm{mV} / \mathrm{N}$, effective mass of $0.1 \mathrm{~kg}$ and maximum force of $4,448 \mathrm{~N}$ was used to strike the blade in the 26 locations depicted in Figure 3. To excite the desired frequency range between $0-550 \mathrm{~Hz}$, a soft polyurethane tip was used. The risk of poor hammer impacts was mitigated through striking each location ten times and averaging the results. The response was measured by two miniature piezoelectric accelerometers model Metra KS94B-100 with an individual weight of $3.2 \mathrm{~g}$, voltage sensitivity of $98.95 \mathrm{mV} / \mathrm{g}$ and operational frequency range of $0.5-28 \mathrm{kHz}$ attached at the tip of the blade (Figure 3). These two accelerometers were attached to the trailing edge (TE) and leading edge (LE) using wax, with only one accelerometer required to measure the response but the other for comparison and redundancy purposes. Force and acceleration readings were taken at a sampling rate of $2,048 \mathrm{~Hz}$ with a measurement time of 80 seconds for each impact. The signals were digitized with a National Instruments (NI) NI-9234 data acquisition card connected to a NI cDAQ-9174 chassis and laptop. NI LabView software was used for signal processing.

\subsection{Experimental modal analysis}

To ensure confidence in the results and for comparison purposes, two system identification algorithms were used to determine the modal parameters of the blade. Accelerance frequency response functions (FRFs) describe the dynamic behavior of a system in terms of a mathematical model, allowing the extraction of dynamic characteristics. The averaged magnitude FRF $(A M F R F)$, similar to the averaged normalized power spectral density [15], was used in this research for quick modal frequency identification using peak picking. The $A M F R F$ at location $i$ is defined as:

$$
\operatorname{AMFRF}_{i}(f)=\frac{\sum_{j=1}^{N_{j}}\left|\mathbf{H}_{i j}(f)\right|}{N_{j}}
$$

with $N_{j}$ equal to the number of excitation locations and $\left|\mathbf{H}_{i j}(f)\right|$ is the $A M F R F$ estimated from numerous measurements at location $j$. Averaging of FRFs in this manner results in a loss of information regarding the local responses, however, it is suitable for determining the global properties of the structure. In addition to $A M F R F$, a subspace identification (SSI) algorithm developed by Overschee and De Moor [16] was used for system identification. 
Return to the Manage Active Submissions page at http://spie.org/submissions/tasks.aspx and approve or disapprove this submission. Your manuscript will not be published without this approval. Please contact author_help@spie.org with any questions or concerns.

Table 1 - Known/measured properties of experimental wind turbine blade

\begin{tabular}{ll} 
Property & Value \\
\hline Length & $2.36 \mathrm{~m}$ \\
Width & $0.16 \mathrm{~m}$ \\
Mass & $7.11 \mathrm{~kg}$ \\
Mass density & $2,300 \mathrm{~kg} / \mathrm{m}^{3}$ \\
Profile & $\mathrm{E} 387$
\end{tabular}

\subsubsection{Experimental modal analysis results in baseline state}

EMA was conducted in the baseline state, without any structural modifications, to provide a target for further updating studies. With no material specifications available from the blades manufacturer, these results will be used to approximate the initial material properties of the blade. The AMFRF obtained from both sensors S1 and S2 (Figure 3) are shown in Figure 4 indicating peaks in the plot at the same natural frequencies. The first eight natural frequencies are provided in

Table 2 with modes 1, 2, 3, 5, 6 and 8 identified as the blades bending modes whilst modes 4 and 7 as torsional vibration modes. The low first natural frequency is indicative of the high flexibility of the blade. The standard deviations associated with each methodology were found to be very low, and near perfect agreement observed between peak picking and SSI, therefore, these results are deemed acceptable.

\subsubsection{Experimental modal analysis results in altered state}

In modelling damage on this blade, consideration had to be given to realistic damage location, realistic damage representation and preventing permanent structural damage to the test specimen. Typical damage locations of full-scale wind turbine blades were investigated by Ataya and Ahmed [17], who carried out visual inspections of ninety nine 100 $\mathrm{kW}$ and $300 \mathrm{~kW}$ blades. The authors noted damage in the form of cracking was most likely to occur at around $73 \%$ blade length and on the TE. This work was in agreement with studies by Ciang et al. [18] which found the TE region, at $72 \%$ length to be highly susceptible to damage. Based on these observations, the location of the structural alteration in this research was chosen to be $70 \%$ length on the TE. To non-destructively simulate damage at this location, a structural alteration through addition of small masses was introduced.

This structural alteration will induce a structural change that can be studied in lieu of damage whilst preventing permanent damage to the structure. To facilitate this, a thin metal plate was glued to the location specified in Figure 3, which enabled the attachment of small magnets. The damage scenario investigated represents addition of $0.405 \mathrm{~kg}$ to the blade edge. The frequencies obtained through the AMFRF methodology can be seen in Table 3 with percentage reduction compared to baseline state highlighted. As shown in Table 3 the effect of added mass vary significantly between $0.4 \%$ and $11.8 \%$, which is of sufficient magnitude to distinguish between the effects of structural alteration and noise and environmental influences in the lab. 
Return to the Manage Active Submissions page at http://spie.org/submissions/tasks.aspx and approve or disapprove this submission. Your manuscript will not be published without this approval. Please contact author_help@spie.org with any questions or concerns.

Table 2- Experimental natural frequencies in baseline state

\begin{tabular}{lllll}
$\begin{array}{l}\text { Mode } \\
\text { no. }\end{array}$ & $\begin{array}{l}\text { AMFRF } \\
\text { frequency } \\
(\mathrm{Hz})\end{array}$ & $\begin{array}{l}\text { Standard } \\
\text { deviation } \\
(\mathrm{Hz})\end{array}$ & $\begin{array}{l}\text { SSI } \\
\text { frequency } \\
(\mathrm{Hz})\end{array}$ & $\begin{array}{l}\text { Standard } \\
\text { deviation } \\
(\mathrm{Hz})\end{array}$ \\
\hline 1 & 1.75 & 0.00 & 1.75 & 0.00 \\
2 & 11.2 & 0.00 & 11.2 & 0.00 \\
3 & 31.3 & 0.00 & 31.3 & 0.00 \\
4 & 38.8 & 0.00 & 38.8 & 0.01 \\
5 & 61.3 & 0.00 & 61.3 & 0.00 \\
6 & 100.4 & 0.01 & 100.5 & 0.00 \\
7 & 116.3 & 0.01 & 116.4 & 0.01 \\
8 & 149.4 & 0.01 & 149.3 & 0.00
\end{tabular}

Table 3 - Comparison of experimental natural frequencies between baseline and damaged state

\begin{tabular}{llll}
$\begin{array}{l}\text { Mode } \\
\text { no. }\end{array}$ & $\begin{array}{l}\text { Baseline } \\
\text { frequency } \\
(\mathrm{Hz})\end{array}$ & $\begin{array}{l}\text { Damaged } \\
\text { frequency } \\
(\mathrm{Hz})\end{array}$ & $\begin{array}{l}\text { Relative } \\
\text { difference } \\
(\%)\end{array}$ \\
\hline 1 & 1.75 & 1.67 & -4.8 \\
2 & 11.2 & 11.1 & -0.7 \\
3 & 31.3 & 29.3 & -6.6 \\
4 & 38.8 & 34.6 & -10.9 \\
5 & 61.3 & 60.7 & -1.0 \\
6 & 100.4 & 99.4 & -1.0 \\
7 & 116.3 & 115.8 & -0.4 \\
8 & 149.4 & 131.8 & -11.8
\end{tabular}

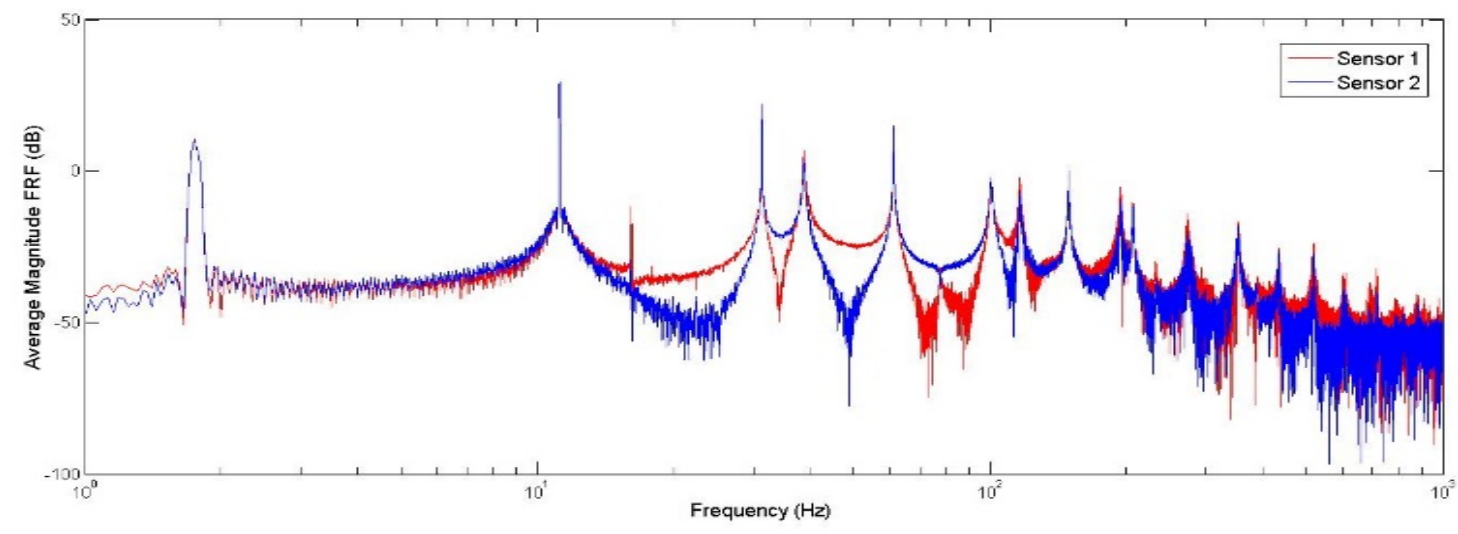

Figure 4 -AMFRF results in baseline state - comparison between two sensors highlighting natural frequencies (peaks) occurring at the same values

\subsection{Fuzzification of experimental results}

To introduce uncertainty into the experimental results, the experimental values obtained in Section 3.2.1 and 3.2.2 were used as inputs to the fuzzification process. The AMFRF values shown in Table 3 were considered deterministic values with membership values equal one $(\alpha=1)$. To account for uncertainty in measurement and system identification, experimental data were modelled as symmetric fuzzy numbers with triangular membership functions. Intervals at level $\alpha=0$ were chosen based on typical measurement error variance and assumed modelling error variance. The measurement error at each $\alpha$-level was defined as [7]:

$$
\Delta_{\alpha, j}^{D}=(1-\alpha) z \sigma_{j}
$$

where $\sigma_{j}$ estimates the standard deviation of measurement error associated with the $j^{\text {th }}$ eigenvalue and $z$ incorporates a confidence interval of errors into the $\alpha$-level. The modelling error at each $\alpha$-level was defined as [7]:

$$
\Delta_{\alpha, j}^{G}=(1-\alpha) z \varepsilon_{j} \lambda_{e j}
$$

where $\varepsilon_{j}$ determines the magnitude of the modelling error. For this investigation, it was assumed based on our own experimental results reported earlier that $\sigma_{j}=0.01 \mathrm{~Hz}, \varepsilon_{j}=0.01$ represents an assumed $1 \%$ modelling error, and $z$ is a function 


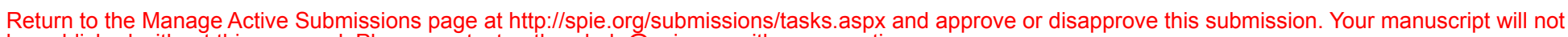
be published without this approval. Please contact author_help@spie.org with any questions or concerns.

of the normal inverse cumulative distribution function. With a $99 \%$ confidence interval chosen the corresponding value was $z=2.58$. The interval bounds were then linearly interpolated to obtain the upper and lower bounds of measured data at each $\alpha$-level as [7]:

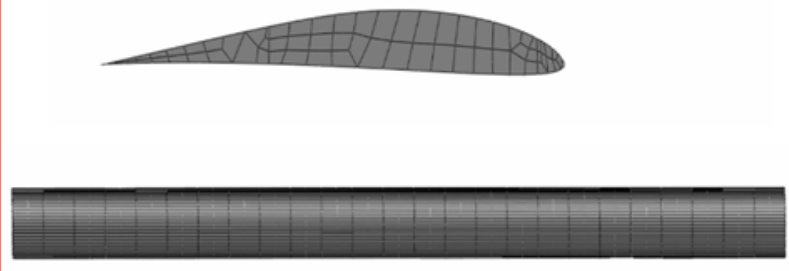

Figure 5 -Blade FEM

$$
\left[\tilde{\lambda}_{j}^{e}\right]_{\alpha}=\left[\lambda_{j}^{e}-\Delta_{\alpha, j}^{D}-\Delta_{\alpha, j}^{G}, \lambda_{j}^{e}+\Delta_{\alpha, j}^{D}+\Delta_{\alpha, j}^{G}\right]
$$

Table 4 - Initial estimate [19] and PSO and FA-updated value of blate material properties

\begin{tabular}{|c|c|c|c|}
\hline Material property & $\begin{array}{c}\text { Initial } \\
\text { estimate }\end{array}$ & $\begin{array}{l}\text { Updated } \\
\text { (PSO) }\end{array}$ & $\begin{array}{c}\text { Updated } \\
\text { (FA) }\end{array}$ \\
\hline $\begin{array}{l}\text { Longitudinal modulus, } E_{1} \\
(\mathrm{GPa})\end{array}$ & 53.48 & 62.14 & 62.15 \\
\hline $\begin{array}{l}\text { Transverse modulus, } E_{2} \\
(\mathrm{GPa})\end{array}$ & 17.7 & 17.7 & 17.7 \\
\hline $\begin{array}{l}\text { In-plane shear modulus, } G_{12} \\
(\mathrm{GPa})\end{array}$ & 5.83 & 8.37 & 8.33 \\
\hline Major Poisson's ratio, $v_{12}$ & 0.278 & 0.278 & 0.278 \\
\hline $\begin{array}{l}\text { Through thickness } \\
\text { Poisson's ratio, } v_{23}\end{array}$ & 0.4 & 0.4 & 0.4 \\
\hline
\end{tabular}

\section{RESULTS}

FEMU is an inverse process using the observed dynamic behavior of a system to determine the model parameters. Although consideration must be given to uncertainty in experimental techniques, it is common practice within FEMU to assume experimental data is accurate and use this data as a target for updating the numerical model. FFEMU breaks from this assumption. This section starts with a description of the numerical model constructed for calibration studies. FFEMU of the model in its baseline (healthy) state is conducted first to obtain an estimate of the blades material properties. Finally, the results of FFEMU for damage identification indicating its location and severity are presented.

\subsection{Numerical model}

A numerical model of the experimental test specimen defined in Section 3.1 was modelled using ABAQUS software as shown in Figure 5. The model consists of 9,828 nodes and 1,775 solid elements. The blade was supported with encastre boundary conditions imposed upon the bottom face to simulate the clamp configuration within the laboratory experiments. An initial estimate of the material properties were obtained from Soden et al. [19] by assuming the blade was an epoxy composite with unidirectional glass fiber reinforcement known as E-glass 21xK43 Gevetex. These material properties, shown in Table 4, were specified in the initial numerical model prior to updating. These properties were updated as shown in Section 3.2.1 to obtain a calibrated baseline FEM.

\subsection{Fuzzy finite element model updating in baseline state}

As discussed in Section 4.1, initial material properties to be used for updating studies can be seen in Table 4, with the longitudinal modulus, $E_{1}$, and in-plane shear modulus, $G_{12}$, chosen as updating parameters. The first stage of fuzzy updating involves the minimization of the deterministic objective function shown in Eq. (2) containing the difference between analytical and experimental eigenvalues. Within this work, two algorithms were utilized for this purpose, namely PSO and FA.

To initialize PSO, a swarm of 10 particles were generated with a maximum number of iterations specified as 50 . A methodology developed by Clerc and Kennedy [20] suggests PSO parameters such as the inertial weight $(\gamma)$ equal to 0.73 
Return to the Manage Active Submissions page at http://spie.org/submissions/tasks.aspx and approve or disapprove this submission. Your manuscript will not be published without this approval. Please contact author_help@spie.org with any questions or concerns.

and the personal/social acceleration coefficients $\left(c_{1} / c_{2}\right)$ as both equal to 1.45 . At each iteration of the PSO algorithm, updated frequency values were obtained using the ABAQUS2Matlab toolbox [13]. The updated deterministic material properties $E_{1}$ and $G_{12}$ obtained through PSO were found to be $62.14 \mathrm{GPa}$ and $8.37 \mathrm{GPa}$, respectively (Table 4). The objective function value calculated for this solution was 0.0357 .

To initialize FA, a population of 10 particles were generated with the maximum number of iterations specified as 10 . The light absorption $(\gamma)$, attraction $\left(\beta_{0}\right)$ and mutation $(\alpha)$ coefficients were specified as 1,2 and 0.2 , respectively. At each iteration of the FA algorithm, updated frequency values were again obtained using the ABAQUS2Matlab toolbox [13]. The updated deterministic material properties $E_{1}$ and $G_{12}$ obtained through FA were found to be $62.15 \mathrm{GPa}$ and $8.33 \mathrm{GPa}$ (Table 4). The objective function value calculated for this solution was 0.0359 .

A comparison between the frequency values obtained for the initial material property specifications and the frequency values obtained for the deterministic levels updated by both PSO and FA can be seen in Table 5. The results from both algorithms show a significant decrease in the maximum percentage variation obtained with this variation decreasing for both algorithms to under $2.5 \%$ for the first mode of vibration. The over error in the first bending mode frequency could be due to the modelling inaccuracies resulting from idealizing the encastre boundary conditions; further study will involve modelling the boundary conditions as springs and including these as updating parameters during model calibration.

In subsequent stages of fuzzy updating, fuzzy experimental results, calculated through the method described in Section 3.3 were used to conduct interval model updating at each individual $\alpha$-level. This was achieved through minimization of the fuzzy objective function shown in Eq. (3) containing the frequency variation at the lower and upper bounds. Using the PSO and FA parameters defined above, the two algorithms were run to obtain interval vectors of updating parameters containing the lower and upper values of updated parameter at each $\alpha$-level. The final fuzzy parameters were obtained by combining the deterministic and interval valued updating parameters at each level as shown in Figure 6. Comparison between the fuzzy updated parameters obtained through both algorithms highlight the coherence between solutions and increase the confidence this solution is in fact the global optimum. A slight difference is noted between the results obtained in the updating of parameter $G_{12}$, however, this is thought to be due to the small number of iterations allowed for the FA algorithm, with the FA algorithm converging on a higher objective function value at each $\alpha$-level. Time permitting, this algorithm will be run again with a greater number of iterations and more detailed investigation into parameters to avoid premature convergence.

Table 5 - Frequency comparison between experimental, initial model, and PSO and FA-updated models in baseline state

\begin{tabular}{|c|c|c|c|c|c|c|c|c|}
\hline Mode no. & 1 & 2 & 3 & 4 & 5 & 6 & 7 & 8 \\
\hline Experimental (Hz) & 1.75 & 11.2 & 31.3 & 38.8 & 61.3 & 100.5 & 116.4 & 149.3 \\
\hline Initial model (Hz) & 1.66 & 10.4 & 29.0 & 32.5 & 56.6 & 92.9 & 99.7 & 138.0 \\
\hline PSO-updated model (Hz) & 1.79 & 11.2 & 31.3 & 38.8 & 61.2 & 100.5 & 117.2 & 149.3 \\
\hline FA-updated model $(\mathrm{Hz})$ & 1.79 & 11.2 & 31.3 & 38.8 & 61.1 & 100.5 & 117.1 & 149.3 \\
\hline Initial model error $(\%)$ & -5.0 & -7.1 & -49.3 & -25.2 & -47.0 & -43.6 & -20.1 & -36.6 \\
\hline PSO-updated model error (\%) & 2.46 & 0.1 & 0.0 & 0.0 & -0.2 & 0.0 & 0.7 & 0.0 \\
\hline FA-updated model error (\%) & 2.44 & 0.1 & 0.0 & -0.1 & -0.2 & 0.0 & 0.7 & 0.0 \\
\hline
\end{tabular}


Return to the Manage Active Submissions page at http://spie.org/submissions/tasks.aspx and approve or disapprove this submission. Your manuscript will not be published without this approval. Please contact author_help@spie.org with any questions or concerns.

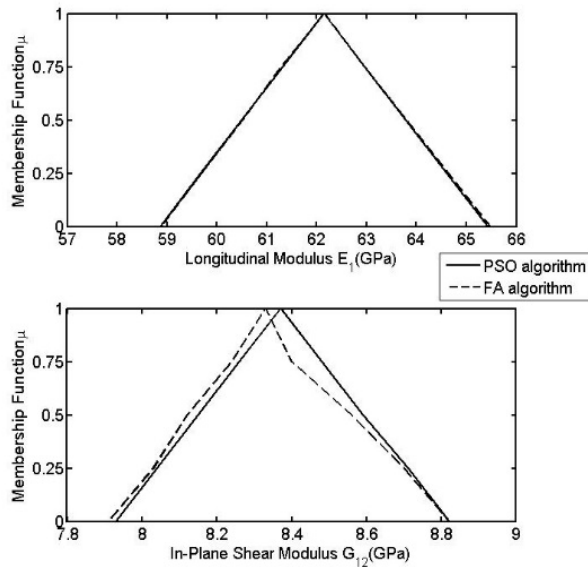

Figure 6 - Fuzzy updated parameters obtained through PSO (black solid line) and FA (dashed line)
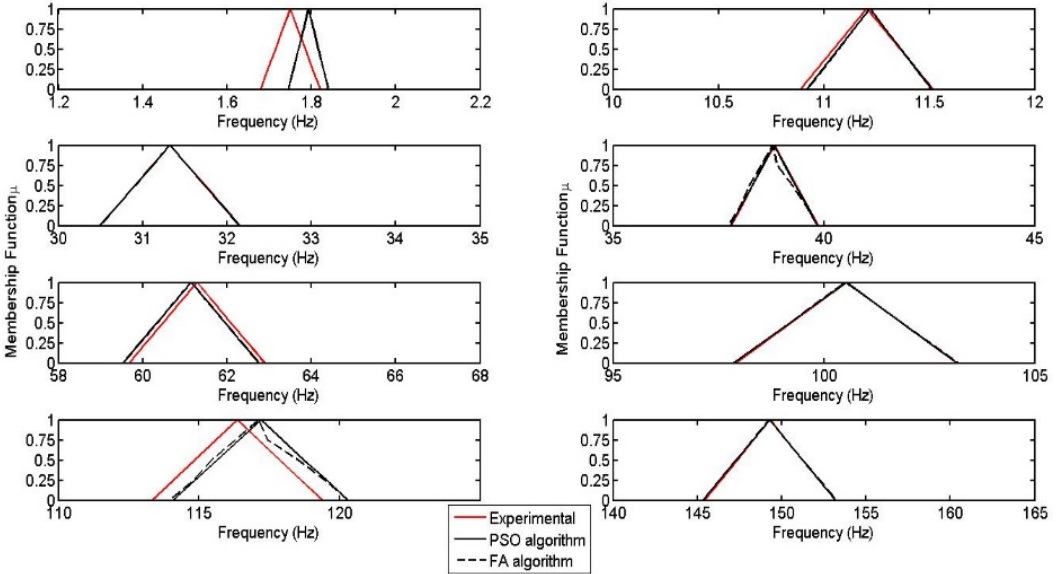

Figure 7 - Comparison between experimental fuzzy frequencies (red solid line) and those obtained through PSO (black solid line) and FA (black dashed line)

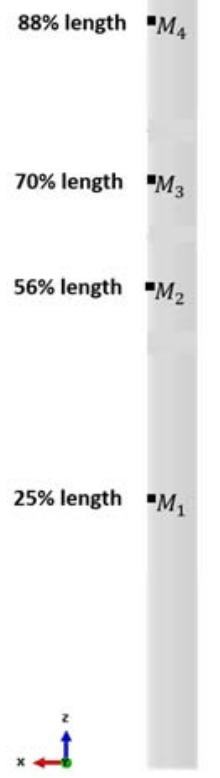

Table 6 - Deterministic damage identification by PSO and FA

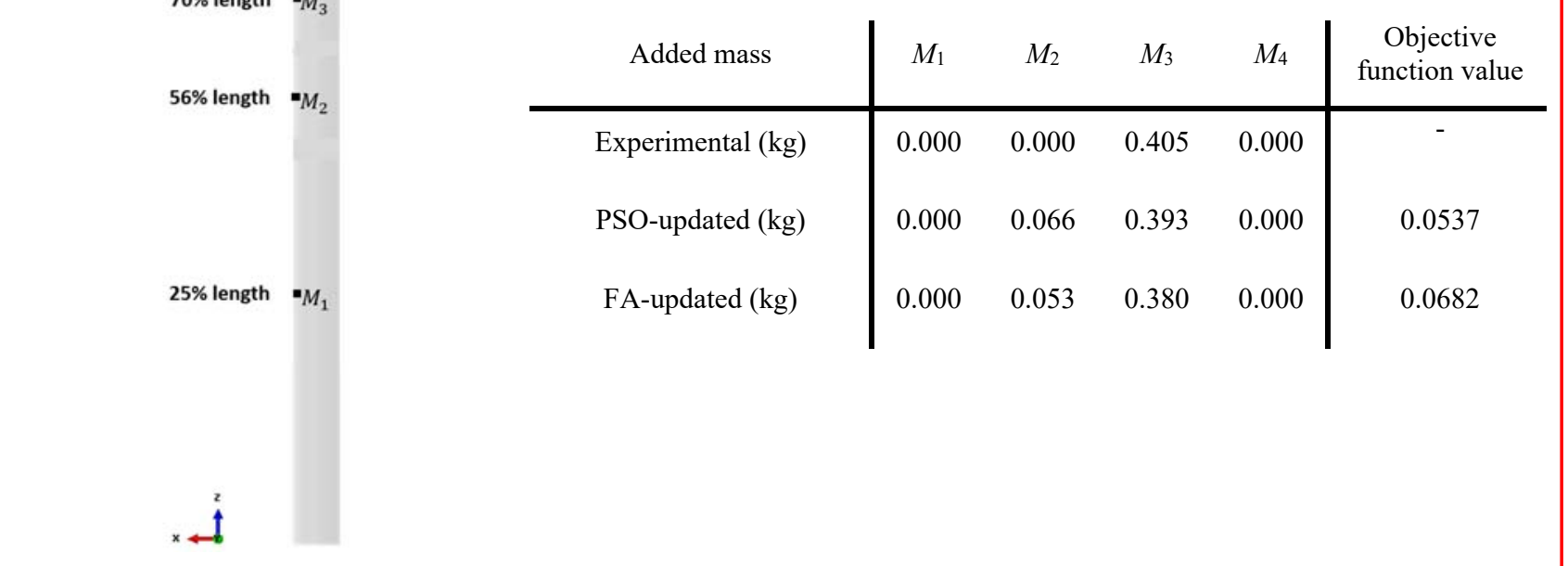

Figure 8 - Blade FEM with added

numerical added masses highlighted.

\subsection{Fuzzy finite element model updating in altered state}

In this section, FFEMU for damage identification is demonstrated with only a singular experimental modification location investigated and natural frequencies of the blade considered as targets for updating. The section begins with the method for simulating experimental damage in the numerical model and progresses onto the updating results obtained via PSO and FA. 
Return to the Manage Active Submissions page at http://spie.org/submissions/tasks.aspx and approve or disapprove this submission. Your manuscript will not be published without this approval. Please contact author_help@spie.org with any questions or concerns.

\subsection{Simulating effects of structural modification}

The updated FEM created in Section 4.2 was utilized in further study to identify magnitude and location of experimentally simulated structural alteration on the blades TE. As discussed in Section 3.2.2, structural alteration was simulated experimentally through addition of mass to the TE of the blade corresponding to a commonly observed damage location, in this case at $70 \%$ length. To locate damage on the numerical model, four masses were modelled at defined points along the TE, as shown in Figure 8. This enabled the mass value of each individual structural alteration to be considered as an updating parameter. With knowledge of experimental alteration location, in addition to common realistic damage locations, it was decided to locate more masses in the 50\%-75\% length region of the blade. This will provide the benefit of accurate comparison between experimental and numerical results.

Updating parameters will be referred to as $M_{n}$ with $n$ denoting the number of the added mass. Updating for damage identification using a similar methodology has been successfully implemented by Behmanesh and Moaveni [4, 5]. Using the process defined in Section 3.3, AMFRF results of the blade in the altered state shown in Table 3 were constructed as fuzzy numbers with triangular membership functions

\subsection{Results of updating with structural alteration}

For the damage identification problem, PSO was initialized with the same parameters as in Section 4.2, however, due to the increased dimensionality of the problem, it was decided to carry out 100 iterations of the algorithm to ensure convergence. Similarly, FA was initialized with the same parameters as above, also increasing the amount of iterations of the algorithm to 30 .

As before, deterministic updating at level $\alpha=1$ was carried out through construction of an objective function and subsequent minimization of this function firstly using PSO and then FA. Updating at the deterministic level for both algorithms indicate a higher magnitude of mass detected at $M_{3}$ with the PSO algorithm predicting this damage with a high level of accuracy (Table 6). Both algorithms performed well, predicting the experimental mass magnitude to within $3 \%$ for PSO and $6.5 \%$ for FA. The deterministic frequency values obtained through both methods can be seen in Table 7 with percentage error from the baseline highlighted.

Table 7 - Frequency comparison between experimental, initial model, and PSO and FA-updated models in altered state

\begin{tabular}{l|cccccccc} 
Mode no. & 1 & 2 & 3 & 4 & 5 & 6 & 7 & 8 \\
\hline Experimental (Hz) & 1.67 & 11.1 & 29.3 & 34.6 & 60.7 & 99.5 & 116.8 & 144.2 \\
PSO-updated model (Hz) & 1.72 & 11.1 & 29.1 & 34.7 & 60.0 & 99.8 & 116.6 & 143.4 \\
FA-updated model (Hz) & 1.72 & 11.1 & 29.3 & 34.5 & 59.9 & 99.6 & 115.3 & 142.6 \\
PSO-updated model error (\%) & 3.2 & 0.0 & -0.1 & 0.3 & -1.0 & 0.1 & 0.0 & -0.7 \\
FA-updated model error (\%) & 3.2 & 0.0 & 0.0 & -0.2 & -1.2 & 0.1 & -1.2 & -1.1
\end{tabular}

As shown in Table 6, the objective function value was lower for the PSO algorithm with future studies focusing on refinement of the FA parameters to improve accuracy. During updating, alteration of a significant magnitude was also detected on segment $M_{2}$ by both algorithms. This could potentially be attributed to issues identified within section 4.2 relating to the large frequency error in updating of the first mode.

Fuzzy updated parameters were constructed through the method described in Section 4.2 with the results acquired through PSO and FA shown in Figure 9 and Figure 10, respectively. The similarity between results obtained by both algorithms is 
Return to the Manage Active Submissions page at http://spie.org/submissions/tasks.aspx and approve or disapprove this submission. Your manuscript will not be published without this approval. Please contact author_help@spie.org with any questions or concerns.

highlighted, but with the FA consistently being less accurately updated, achieving higher objective function values at each level shown in

Table 8. A comparison between the experimental and updated fuzzy frequency values obtained can be seen in Figure 11. As shown both algorithms are in relatively good agreement through all 8 modes, however for mode 1, 5 and 7 the frequency values vary noticeably from the experimental values. This issue could potentially be caused by the slightly worse updating outcome for these modes within the baseline study described in section 4.2 and Figure 7.

The ability to detect magnitude of structural alteration was successful demonstrated using both algorithms with PSO consistently providing a superior accuracy. Future research will involve refinement of the numerical model created and investigation of the algorithms used for updating.
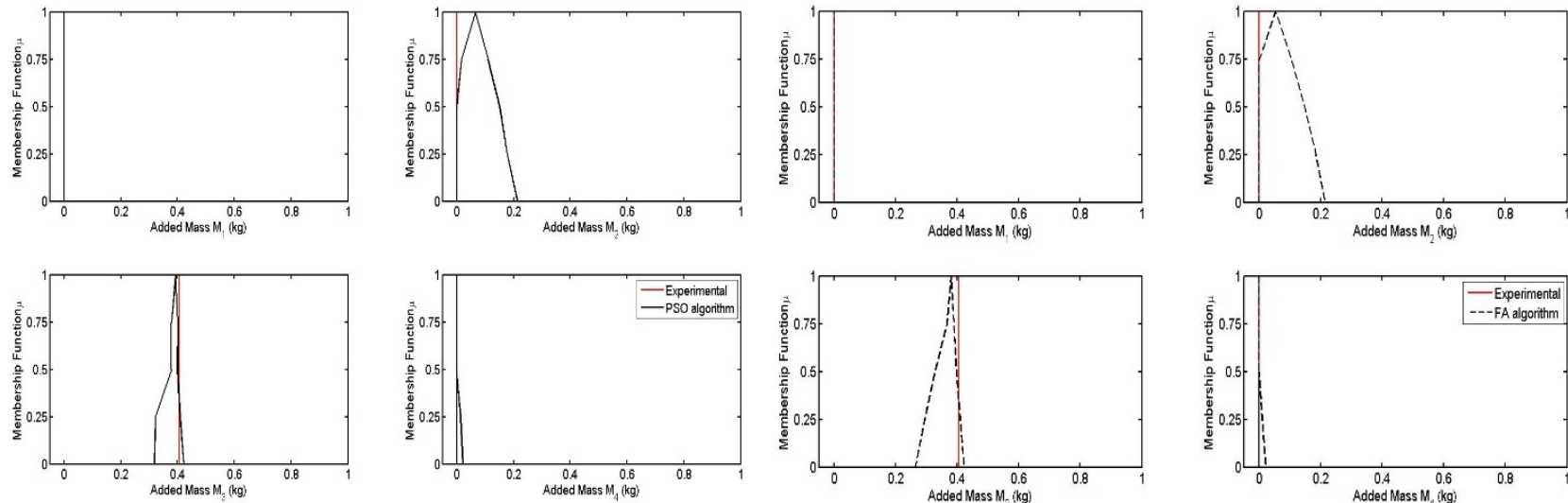

Figure 9 - PSO-updated fuzzy mass (black) and actual experimental mass (red)
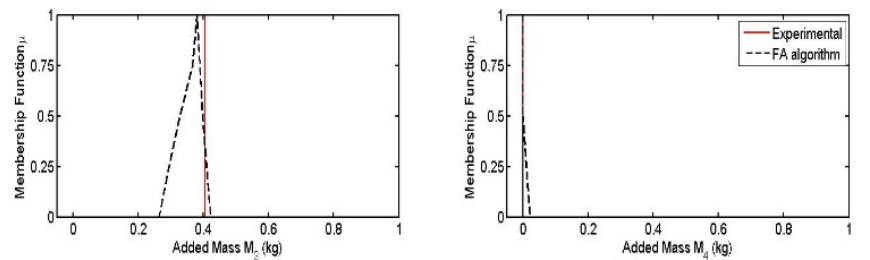

Figure 10 - FA-updated fuzzy mass (black) and actual experimental mass (red)

Table 8 - Objective function values obtained by PSO and FA

\begin{tabular}{c|cc}
$\begin{array}{c}\text { Membership } \\
\text { Function } \\
\text { Value }\end{array}$ & $\begin{array}{c}\text { PSO } \\
\text { Objective } \\
\text { Value }\end{array}$ & $\begin{array}{c}\text { FA } \\
\text { Objective } \\
\text { Value }\end{array}$ \\
\hline 1 & 0.0537 & 0.0682 \\
0.75 & 0.1193 & 0.1440 \\
0.5 & 0.1305 & 0.1582 \\
0.25 & 0.1492 & 0.1746 \\
0 & 0.1555 & 0.1968
\end{tabular}
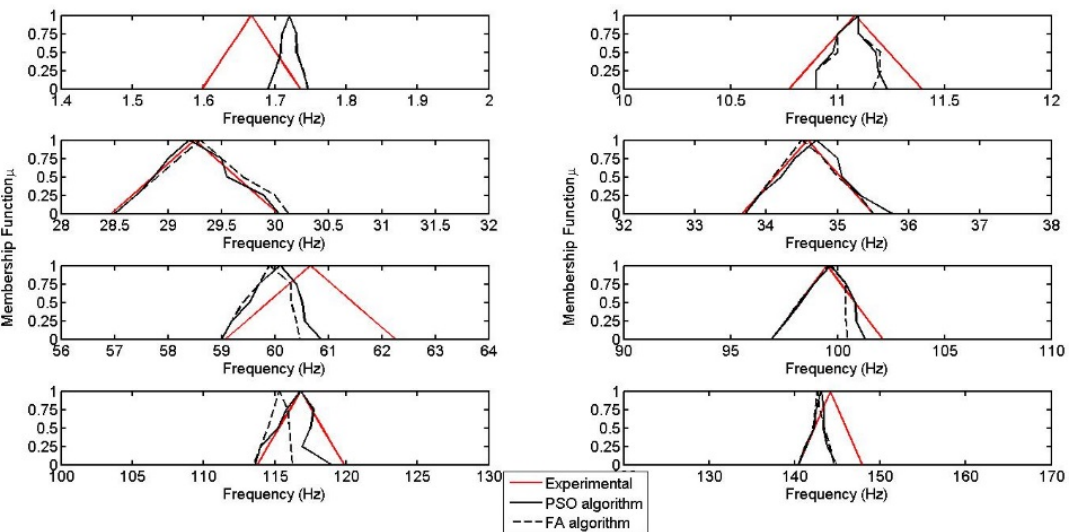

Figure 11 - Experimental fuzzy frequencies (red solid line) and obtained through PSO (black solid line) and FA (black dashed line)

\section{CONCLUSIONS}

In this research, FFEMU was discussed and implemented to update a small-scale wind turbine blade in laboratory conditions. The numerical model was updated using the experimental results obtained from EMA in both baseline and altered states. Updating the model in its baseline state (no added mass) provided a realistic estimate of the material properties to be used in further damage estimation studies. FFEMU was then utilized to detect location and predict magnitude of structural alteration (added mass) with fuzzy updated parameters constructed accounting for the uncertainty within the updating parameters. This methodology was able to predict a more significant magnitude of structural alteration 


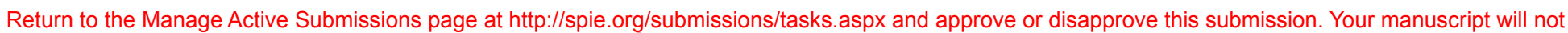
be published without this approval. Please contact author_help@spie.org with any questions or concerns.

on the region where it was actually located using both algorithms. Further refinement of the numerical model and algorithms used for updating will be the focus of further research.

\section{ACKNOWLEDGMENT}

Heather Turnbull's PhD study within the Lloyd's Register Foundation Centre for Safety and Reliability Engineering at the University of Aberdeen is supported by Lloyd's Register Foundation. The Foundation helps to protect life and property by supporting engineering-related education, public engagement and the application of research.

\section{REFERENCES}

[1] Global Wind Energy Council (GWEC), (2016). Global wind report annual market update 2015, Available at: http://www.gwec.net/global-figures/wind-energy-global-status/

[2] Fischer, K., Besnard, F. and Bertling, L., (2012). Reliability-centered maintenance for wind turbines based on statistical analysis and practical experience, Energy Conversion, IEEE Transactions On, 27 (1), pp.184-195.

[3] Mottershead, J.E., Link, M. and Friswell, M.I., (2011). The sensitivity method in finite element model updating: A tutorial, Mechanical Systems and Signal Processing, 25 (7), pp.2275-2296.

[4] Behmanesh, I. and Moaveni, B., (2015). Probabilistic identification of simulated damage on the Dowling Hall Footbridge through Bayesian finite element model updating, Structural Control and Health Monitoring, 22 (3), pp.463-483.

[5] Behmanesh, I. and Moaveni, B., (2016). Accounting for environmental variability, modeling errors, and parameter estimation uncertainties in structural identification, Journal of Sound and Vibration, 374 pp.92-110.

[6] Chandrashekhar, M. and Ganguli, R., (2009). Uncertainty handling in structural damage detection using fuzzy logic and probabilistic simulation, Mechanical Systems and Signal Processing, 23 (2), pp.384-404.

[7] Simoen, E., De Roeck, G. and Lombaert, G., (2015). Dealing with uncertainty in model updating for damage assessment: A review, Mechanical Systems and Signal Processing, 56 pp.123-149.

[8] Erdogan, Y.S. and Bakir, P.G., (2013). Inverse propagation of uncertainties in finite element model updating through use of fuzzy arithmetic, Engineering Applications of Artificial Intelligence, 26 (1), pp.357-367.

[9] Liu, Y. and Duan, Z., (2012). Fuzzy finite element model updating of bridges by considering the uncertainty of the measured modal parameters, Science China Technological Sciences, 55 (11), pp.3109-3117.

[10] Zadeh, L.A., (1965). Fuzzy sets, Information and Control, 8 (3), pp.338-353.

[11] Shabbir, F. and Omenzetter, P., (2015). Particle swarm optimization with sequential niche technique for dynamic finite element model updating, Computer-Aided Civil and Infrastructure Engineering, 30 (5), pp.359-375.

[12] Yang, X.S., (2008). Firefly algorithm, in Nature-Inspired Metaheuristic Algorithms, 2nd ed. pp. 79.

[13] Fister, I., Fister Jr., I., Yang, X. and Brest, J., (2013). A comprehensive review of firefly algorithms, Swarm and Evolutionary Computation, 13, pp.34-46.

[14] Kennedy, J. and Eberhart, R., (1995). Particle swarm optimization, pp. 1942-1948.

[15] Felber, A.J. and Ventura, C.E., (1996). Frequency domain analysis of the ambient vibration data of the Queensborough bridge main span, Proceedings of 14th International Modal Analysis Conference (IMAC), Dearborn, pp. 459-465.

[16] Overschee, P.V. and De Moor, B., (1996). Subspace identification for linear systems, Massachusetts, US: Kluwer Academic Publishers.

[17] Ataya, S. and Ahmed, M.M.Z., (2013). Damages of wind turbine blade trailing edge: Forms, location, and root causes, Engineering Failure Analysis, 35 pp.480-488.

[18] Ciang, C.C., Lee, J. and Bang, H., (2008). Structural health monitoring for a wind turbine system: A review of damage detection methods, Measurement Science \& Technology, 19 (12),

[19] Soden, P.D., Hinton, M.J. and Kaddour, A.S., (1998). Lamina properties, lay-up configurations and loading conditions for a range of fibre-reinforced composite laminates, Composites Science and Technology, 58 (7), pp.1011-1022.

[20] M. Clerc and J. Kennedy, (2002). The particle swarm - explosion, stability, and convergence in a multidimensional complex space, IEEE Transactions on Evolutionary Computation, 6 (1), pp.58-73. 